\title{
La violencia doméstica como fenómeno invisibilizado
}

Diana Gladys Barimboim ${ }^{1}$

UADE

\section{Ensayo}

Material original autorizado para su primera publicación en el Journal de Ciencias Sociales, Revista Académica de la Facultad de Ciencias Sociales de la Universidad de Palermo.

\section{Recibido: 2-6-2017}

Aceptado: 13-1-2017

Resumen: El presente ensayo es producto de las reflexiones realizadas a partir de una investigación descriptiva realizada en el INSOD (UADE) sobre violencia de género femenino a masculino en AMBA. A partir de allí, consideramos que la violencia doméstica es un fenómeno invisibilizado, ya que es privativo de las familias y que sus miembros lo naturalizan como único modo de vincularse.

Consideramos importante la visibilización social que se ha hecho en los últimos años de la violencia de género masculino hacia el femenino, pero consideramos que, en el ámbito de la intimidad familiar, donde los vínculos debieran ser fuente de seguridad y apego, se pueden jugar los mayores sufrimientos subjetivos. Este fenómeno no es privativo del género femenino, sino que observamos que también el género masculino y los niños pueden padecer violencia psicológica (que es el tema del que tratamos en este ensayo).

Resulta difícil hacer público lo privado en estas circunstancias, ya que las víctimas tienen temor de perder el amor del victimario que también es fuente de amor y seguridad. Además, se produce en ellas, justamente por la ambivalencia del vínculo, un sentimiento de culpa que los hace muchas veces sentir que merecen el castigo propiciado por el miembro de la familia que tiene un lugar de autoridad. Desde ya que la violencia siempre se desarrolla en vínculos asimétricos, donde uno de los polos se cree con derecho de imponer sus creencias y formas de actuar.

Pensamos los actos de violencia familiar a partir del concepto de violencia secundaria desarrollados por Piera Aulagnier (1997). El violentador ejerce un poder extremo que conlleva la destrucción de la subjetividad de la víctima.

\footnotetext{
${ }^{1}$ Doctora en Psicología Social. Psicoanalista. Docente en la Universidad Argentina de la Empresa. Correo electrónico: diana@barimboim.com
} 
Ofrecer un apoyo desde el contexto social, sería una de las maneras de legitimar este tipo de maltrato y ayudar a las víctimas a denunciarlo para protegerse.

Palabras Clave: Violencia doméstica, vínculo asimétrico, género, invisibilidad social.

Abstract: This essay is the result of the reflections from a descriptive research in the INSOD (UADE) on gender violence feminine masculine in AMBA. From there we consider that domestic violence is an invisible phenomenon, as it is exclusive to families and their members naturalize him as the only way to link. There we consider that domestic violence is an invisible phenomenon, as it is exclusive to families and their members naturalize him as the only way to link.

We consider important social visibility that has been made in recent violence of male to female, but we believe that in the field of family privacy, where the links should be a source of security and attachment, you can play the greatest sufferings subjective. This phenomenon is not exclusive to the female gender but also observed that the male gender and children can suffer psychological violence (which is the subject discussed in this essay).

It is difficult public to private in these circumstances because victims are afraid of losing the love of the perpetrator who is also a source of love and security. In addition, it occurs in them, precisely because of the ambivalence of the bond, a sense of guilt that makes many feel they deserve the punishment sometimes led by the member of the family that has a place of authority. Since violence always develops in asymmetric links where one pole is created with the right to impose their beliefs and ways of acting.

These acts of violence on victims pierce their own self-esteem to negating the possibility of thought, as developed Piera Aulagnier (1997) through the concept of school violence.

Provide support from the social context, it would be one way of legitimizing this kind of abuse and help victims to report it to protect themselves.

Key words: Domestic violence, asymmetrical link, gender, social invisibility.

\section{Introducción}

Este ensayo es una reflexión sobre la violencia familiar que, a pesar de ser un fenómeno invisibilizado en la sociedad, consideramos muy importante develarlo por sus consecuencias subjetivas y sociales. Han tenido que 
transcurrir años para que se comience a hablar de la violencia de género. Con el advenimiento del movimiento feminista, se comenzó a visualizar una conducta que parecía naturalizada en la sociedad occidental y patriarcal: la violencia del género masculino al femenino.

Al hablar de violencia de género, tanto en los ámbitos científicos como desde el sentido común, se ubica al varón como victimario y a la mujer como víctima.

En verdad, la violencia en los ámbitos de la intimidad (familiar) no es registrada por sus integrantes, ya que pareciera ser un modo "normal" de comunicación y trato dentro de los miembros que habitan familias disfuncionales. Este fenómeno, que pertenece a la vida cotidiana, como tantos otros, es naturalizado también por los miembros de la sociedad. Desde el Instituto de Ciencias Sociales y Proyectuales de la Fundación UADE realizamos una investigación descriptiva desde el marco de la psicología social crítica y el psicoanálisis, para detectar la violencia psicológica que las mujeres realizan sobre los hombres en los ámbitos tanto familiares como laborales. La preocupación surge a partir de la observación clínica del incremento de este fenómeno, aunque hasta el momento hay mayor cantidad de denuncias legales realizadas del género masculino al femenino por la visibilidad social que se le ha dado a este fenómeno en los últimos años. También apuntamos a investigar sobre la violencia que ejercen los padres respecto de sus hijos.

La violencia es una expresión de la agresividad humana que intenta destruir abusivamente al otro desde un lugar de poder. No tomamos como eje de este ensayo la violencia física sino la psicológica, por lo que en este último caso no hay una destrucción del cuerpo del otro, sino que se lo intenta someter, subyugar, es decir, no se tolera la diferencia subjetiva. La violencia doméstica no es algo propio de la época en que vivimos, lo que surge como novedoso es que a partir del ideal de "libertad" forjado en la posmodernidad, el acatamiento a la autoridad es cuestionado socialmente. Sin embargo, pensamos que, así como la pulsión de vida y muerte actúan dialécticamente en el psiquismo, la ambivalencia es condición del afecto, y todo vínculo transita entre Narciso y Edipo, la violencia no se ha erradicado del mundo ni tampoco del seno familiar. La diferencia sustancial con otras épocas es que, en la actual, los fenómenos de violencia tratan de ser silenciados, porque socialmente hay un mandato que la censura, en pos del ideal de libertad.

Al hablar de violencia familiar no nos referimos a un hecho aislado, sino a un patrón de conducta que se repite en la pareja y se replica en alguno o en todos los hijos. La violencia psicológica es aquella que se expresa a través de palabras humillantes, reproches, o actos (que a veces están representados por largos silencios o indiferencia). Estas conductas apuntan a menoscabar la autoestima de la víctima, sencillamente por no "ser o no hacer" lo que especularmente desea el otro, quien se posiciona en un lugar asimétrico de poder. Este tipo de violencia es lacerante, ya que se dirige al "ser" de la víctima, quien otorga a la palabra del victimario un lugar de autoridad y, por lo tanto, de 
verdad. Esto hace difícil salir del circuito, ya que la víctima suele terminar sintiéndose culpable por no responder a la demanda del otro, con temor a perder su amor.

El diseño metodológico propuesto para esta investigación recupera la modalidad de triangulación metodológica, con la pretensión de alcanzar mayor certidumbre en las inferencias inductivas realizadas. En este sentido, se pretendió combinar técnicas de recolección del enfoque cuantitativo y del diseño cualitativo. A partir de la propuesta de Jick (2004), se realizó una perspectiva multimétodo con el propósito de profundizar en las dimensiones de análisis propuestas en el diseño original. La triangulación fue intra-método. Desde la perspectiva inductivista, se propuso el trabajo con técnicas de recolección de datos de ambas perspectivas metodológicas. Para el primer enfoque, se previó el diseño y la administración de encuestas auto-administradas con preguntas de opción múltiple y escalas actitudinales producidas ad hoc. Las encuestas fueron realizadas por el equipo de investigación, que supervisó el trabajo de campo a partir de la confirmación de un adecuado llenado de las encuestas auto-administradas. Se previó tanto el diseño de escalas de Lickert ad hoc, como la implementación de las escalas actitudinales de medición de violencia de género de comprobada confiabilidad (Araya Martínez, 2011; Valdez y otros, 2006). La escala para medir creencias que perpetúan la violencia (Araya Martínez, 2011) cuenta con un nivel de confianza satisfactorio, obtenida a partir del alpha de Crombach, con un nivel de consistencia de 0,81. En el caso de la escala para medir violencia e índice de severidad (Valdez y otros, 2006), el nivel de confianza es muy alto, en tanto que los resultados obtenidos por medio de la misma arrojan un alpha de Crombach cuyo nivel de consistencia interna se estima en 0,99. Para ello, se elaboró una muestra no probabilística por cuotas de un tamaño de 400 casos. El criterio de selección de la muestra fue el establecido en el diseño original: hombres y mujeres de clase media /alta en AMBA (según datos INDEC), segmentados en dos grupos de edades: 25 a 35 años y de 50 a 60 años. Las cuotas permitieron alcanzar un valor idéntico para cada subgrupo (a partir de la variable edad-género). Para el desarrollo de la perspectiva cualitativa, se diseñó un guión de entrevista semi-estructurado. Las entrevistas fueron administradas a una muestra no probabilística conformada a partir de la técnica de bola de nieve. El tamaño de la muestra fue de 10 entrevistas con el criterio de sujeción a la instancia de saturación teórica (Glaser y Strauss, 1967). Las entrevistas fueron realizadas por los miembros del equipo de investigación, promoviendo la "triangulación de observadores" (Maxwell, 2014). Las mismas fueron realizadas según los usos y costumbres de la perspectiva cualitativa (contrato de entrevista, grabación de las mismas, desgrabación y posterior codificación con sistema Atlas Ti). Si bien se privilegió la aparición de dimensiones emergentes en el proceso de entrevista, se contó con un guion previo, el cual se indica al final del documento. Las técnicas de análisis de datos incluyeron tanto el análisis estadístico de los datos cuantitativos como el análisis de contenido de los datos cualitativos, de tal modo que el desarrollo del trabajo de campo revele la existencia de dimensiones emergentes de análisis no contempladas en el diseño original del proyecto. En este sentido, se siguió la propuesta teórico-metodológica de Glaser y Strauss, quienes sostienen que la teoría fundada en los datos habilita la posibilidad de identificar tales dimensiones e incorporarlas en el proyecto original. 


\section{Entrevista semidirigida violencia de género}

1) ¿Pensás que existe la violencia de la mujer hacia el hombre? ¿O la violencia siempre es del hombre hacia la mujer?

2) ¿Por qué creés que las mujeres agreden a su pareja? ¿Cómo lo hacen? Pensá en las distintas posibilidades que se pueden considerar violencia.

3) ¿Cuál creés que es la respuesta del hombre frente a las agresiones de la mujer?

4) ¿Por qué creés que los hombres agreden a las mujeres? ¿Cómo lo hacen?

5) ¿Creés que de vez en cuando para que los chicos obedezcan hay que pegarles un chirlo? ¿La autoridad de los padres debe manifestarse eventualmente por medio de algún chirlo?

6) ¿Creés que la violencia femenina se incrementó en estos últimos años? ¿Cómo? ¿Por qué?

7) Cuando la mujer gana más dinero que el hombre, ¿considerás que tiene más poder en la relación con respecto a las decisiones?

8) ¿Cómo reaccionan las mujeres cuando los hombres ponen límites a los hijos? ¿Y cuando son permisivos? ¿Creés que, si hay desacuerdo entre los padres con relación a esto, los chicos se dan cuenta?

9) ¿Cómo son las mujeres como jefas cuando el subalterno es un hombre?

10) Frente a una separación conyugal, ¿quién tiene el poder y la autoridad sobre los hijos? ¿Quién fija horarios de visita? ¿Quién se hace cargo de los niños?

11) ¿Cuáles son las demandas más frecuentes de las mujeres hacia el hombre?

12) ¿En qué áreas se presentan las demandas de las mujeres hacia los hombres? ¿En el sexo? ¿En la economía doméstica? ¿En el cuidado de los chicos?

Se realizaron entrevistas semi-dirigidas y 400 encuestas a hombres y mujeres de clase media/alta (convivientes o casados) del Area Metropolitana de Buenos Aires.

\section{Argumentación teórica}

Al pensar en la violencia doméstica hablamos del ejercicio de poder de uno o más miembros de la familia sobre otro/s al que fundamentalmente se lo convierte en objeto, impidiéndole ser sujeto de deseo y sumiéndolo en una pérdida de identidad propia. Dentro del Psicoanálisis, Piera Aulagnier (1997) conceptualiza la violencia primaria 
como necesaria y constitutiva del sujeto, y la violencia secundaria como un exceso de poder que impide el pensamiento propio y el sostenimiento del yo. Los vínculos familiares, por su misma función, resultan privilegiados para hacer jugar el narcisismo que implica la homogeneización entre los miembros. Es decir, que son familias que se creen con el derecho de imponer sus modelos transfamiliares al otro, como único discurso "verdadero". Esto hace que algunos de sus miembros padezcan un proceso de desubjetivación y tengan trastornos severos en la construcción del propio pensamiento.

La violencia doméstica psicológica es difícil de investigar, dado que el hogar es el lugar identificado como el ámbito de seguridad, sostén y amor. Por este motivo subjetivamente es conflictivo aceptar que, en ese mismo lugar, se pueden vivir los actos de violencia más extremos y a veces, el lugar más temido e inseguro para el sujeto. Esta dificultad para metabolizar psicológicamente dichos actos son los que los tornan traumáticos; resultan un cúmulo de estímulos que amenazan la integridad del sujeto. Hay una creencia sostenida socialmente que idealiza al hogar como un lugar de amor y contención que dista mucho de lo que sucede en los vínculos intersubjetivos íntimos. Surgen relaciones de rivalidad, competencia, deseos de posesión del objeto amado, es decir, sentimientos y situaciones de fuerte ambivalencia, que se alejan de la representación social del "hogar, dulce hogar". Con el develamiento de la violencia de género, últimamente la sociedad está pudiendo pensar que el lugar donde debieran jugarse los sentimientos más tiernos también puede ser el lugar de las luchas más violentas y crueles.

La violencia doméstica en general, es decir en el ámbito familiar, puede incluir los vínculos parento-filiales, los de la pareja y también los filiales. Cuando nos referimos a ella estamos significando una relación asimétrica, donde uno de los ejes del vínculo asume y se le adjudica un lugar de poder, e intenta mediante la violencia tanto física como psicológica desconocer, no tolerar las diferencias individuales, y negar todo respeto por la singularidad del otro.

Muchas veces el acto violento es disfrazado por un "acto de amor" dentro de las relaciones íntimas. La frase vulgar de "lo hago por tu bien" implica un acto de posesión en una relación asimétrica que intenta marcar una situación de poder de un miembro sobre otro, anulando hasta su propia capacidad de pensamiento. En general la persona que ejerce la violencia es muy exigente y tiene ideales muy rígidos sobre su comportamiento y el de los otros miembros. Lo que intenta con sus actos de violencia psicológica, descalificación, y amenazas es que el otro cumpla con sus expectativas de perfección, sistema de creencias e ideales.

Hablamos de violencia psicológica en la pareja cuando hay conductas de menosprecio, burla, hostigamiento, etc. Tomamos en esta investigación la violencia de género en relaciones heterosexuales, es decir, al conjunto de conductas que, desde el orden cultural, se espera que un sujeto desempeñe, a través del proceso de sexuación que realizara desde antes del nacimiento, en su desarrollo evolutivo, a partir de identificaciones con modelos familiares, sociales y culturales. Sabemos que el cambio producido en las últimas décadas de los roles del género femenino 
(definida en la época patriarcal como el negativo del género masculino), está generando un nuevo paradigma en las relaciones interpersonales. El género es una construcción que se realiza a lo largo del ciclo vital en un contexto intersubjetivo. Es decir que no hablamos del sexo biológico del que se ocupan las ciencias médicas, sino de la sexuación subjetiva.

¿Qué le pasa emocional y cognitivamente al hombre con el cambio que realizó la mujer? ¿Cómo juega su identidad masculina con las nuevas exigencias en su rol de padre y pareja? Su lugar de protector, proveedor económico de la familia, etc. es ahora compartido con su pareja. ¿Cómo se posiciona la mujer en el vínculo con el varón, a partir de su independencia económica? ¿Cuáles son las expectativas de la mujer que desarrolla una actividad profesional, en el ámbito familiar? El 53\% de la muestra opina que los hombres y las mujeres se encuentran en igualdad de condiciones. ¿Se válida esto en las prácticas hogareñas cotidianas?

Al hombre se le exige un rol más activo en el cuidado de los hijos y participación en las tareas domésticas. Los estereotipos forjados durante el patriarcado son matrices de aprendizaje vigentes todavía en el psiquismo y hasta sostenidos por los medios de comunicación. Observamos por ejemplo que hasta desde la publicidad se siguen utilizando modelos femeninos para el consumo de artículos relacionados con la higiene del hogar, la mujer sigue siendo utilizada como objeto sexual masculino, etc. A los varones no se les compra muñecas para jugar, y la mayoría de los muñecos que se producen para la venta son niñas. Sin embargo, llegada la edad adulta, se espera que los hombres se hagan cargo de las tareas domésticas y del cuidado de sus hijos.

El género masculino se encuentra en un conflicto identitario entre las viejas matrices de aprendizaje que generaron los estereotipos de masculinidad y las nuevas exigencias socioculturales que implican una simetría en los roles domésticos y parentales. El género femenino, que ancestralmente ha tenido el dominio sobre el hogar y los hijos, ahora desea compartirlo. La mujer, identificada con el rol de ama de casa y madre, se hacía cargo de sostener las cuestiones del hogar. El padre, en cambio, representaba la figura de autoridad para los hijos. En la actualidad, el $28 \%$ de los encuestados plantea que el padre no es la máxima autoridad de la familia y el $15 \%$ plantea que en un divorcio, el padre se encuentra en inferioridad de condiciones respecto de la madre con relación a sus hijos.

En la era patriarcal los roles de género tenían una división binaria, la mujer era el negativo del hombre, así el varón tenia el poder sobre el "sexo débil" a partir de lo económico, su fuerza física y su lugar de reconocimiento en la sociedad. La mujer, si bien permanecía "guardada" dentro del ámbito familiar, allí era el "ama" (dueña) de la casa y de los niños. En los tiempos que corren, la mujer al salir al mercado laboral se ha vuelto "fuerte" (el $18 \%$ de nuestros encuestados adhieren a la idea que poder procurarse su sustento económico les da poder e independencia del hombre). Esta mujer "fuerte" que tiene muchas menos horas para cuidar de sus hijos y de su casa, requiere de su pareja una colaboración equitativa en todas las tareas. 
Sin embargo, sabemos que en esta división de antaño la mujer tenía cierto poder sobre el manejo tanto de los hijos como de la casa. Los hombres, cuando niños, no han aprendido a partir del proceso de socialización a ejercer los roles que hoy le son exigidos. La mujer quiere colaboración, pero sin perder el dominio, que ancestralmente ha tenido, sobre los hijos (que le "pertenecen"). Así es como si bien reclama participación al 50\% del género masculino, también impone sus formas, sin permitir que el género masculino haga las cosas a su manera. En una entrevista, un hombre se quejaba porque su mujer le criticaba "su forma de barrer la casa". Tampoco acuerdan en la educación de los hijos y allí se observa que descalifican y reprochan el ejercicio del rol paterno. En la muestra, el $17 \%$ de los encuestados dice que la mujer trata de imponer su voluntad frente a la de su pareja y el $18 \%$ afirma que las mujeres provocan al género masculino para obtener lo que quieren.

Desde el psicoanálisis sabemos que todos los sentimientos del ser humano son ambivalentes, siendo el odio muchas veces reprimido en las relaciones amorosas. Sin embargo, cuando se generan necesidades insatisfechas, sentimientos de abandono, incomunicación, etc. surgen fantasías de venganza, posesión y deseo de sometimiento hacia el otro, lo que lleva a situaciones de maltrato (Berenstein, 1991). En la convivencia familiar se despliega el afán destructor más fuerte, debido a la intensidad de las relaciones pasionales o de amor narcisista. Esto no tiene que ver con clases sociales/económicas ni culturales. De hecho, las estadísticas muestran que países desarrollados como Finlandia tienen un 10,32 de asesinos por cada millón de mujeres de más de 14 años. (http://www.ine.es).

En las relaciones de pareja se proyectan en el otro las propias dificultades. La vida en pareja, así como la paternidad y la maternidad, significan ciertas renuncias a proyectos personales. Esta interdependencia amorosa es vivida como limitante de la propia individualidad, y a veces se trastoca en una serie de reproches y maltrato al otro. Pensando en lo intersubjetivo, la violencia es ejercida por quien intenta transformar al otro tratando de tornarlo idéntico a sí mismo. Desde ese lugar, borra su singularidad, se vive una cierta ajenidad por lo que hace justamente al otro, "otro". Se trata de "normalizar" al otro, es decir, someterlo a su propio sistema de creencias y normas consetudinarias. El 37\% afirma que las discusiones son originadas en mayor medida por la mujer porque quiere imponer al hombre su forma de pensar y hacer respecto a la pareja, a los hijos y a las tareas domésticas.

Los hombres silencian la violencia que padecen de sus parejas, por vergüenza, machismo, orgullo, temor a ser ridiculizado socialmente. El 22\% de los encuestados afirma que el hombre que tolera violencia de parte de su mujer debe tener un problema psicológico. Este es uno de los motivos por los que el fenómeno descripto aparece invisibilizado socialmente. Denunciar el menoscabo que una mujer puede hacerle a un hombre es de "poco hombre", sería para el género masculino como ir en contra del estereotipo machista aprendido como matriz desde su primera infancia. Un entrevistado comenta que la "tolerancia por amor a la mujer o a la familia, significa ser un pollerudo". El estereotipo de la mujer como sexo débil prima como representación social, haciendo que el hombre generalmente se sienta culpable frente a las demandas, muchas veces violentas, de la pareja. 
La mujer, aunque haya producido cambios que la benefician en relación con la equidad de género, guarda todavía estereotipos machistas que gobiernan ciertas expectativas con relación a la pareja y la familia. Así, aquella mujer que ha logrado un cierto bienestar económico y se ha tornado independiente (independencia que la deja sin apoyo ni sostén por parte del hombre), lo maltrata y descalifica, y siente que ella podría sostener una familia sola, sin el género masculino. Cuando el hombre no cumple con las expectativas femeninas, la mujer horada su autoestima a partir de menoscabar el desempeño de sus roles en el hogar y respecto de sus hijos, mientras continúa exigiendo que cumpla un rol protector como antaño. Cuando el hombre no cumple sus ideales, la mujer siente que puede prescindir de él y genera situaciones de mucho maltrato y hasta situaciones de infidelidad.

El ideal de autosuficiencia tanto social como subjetivo otrora correspondía al género masculino. Los cambios producidos en la mujer, fundamentalmente en el área profesional (lo que conlleva a su posición económica independiente) hace que en nuestros días el lugar de poder en la pareja lo encarne el género femenino. Dicho ideal la posiciona tanto social como subjetivamente en un lugar de dominio y control de las relaciones vinculares, generando conductas de maltrato hacia su partenaire.

La base del maltrato es la ira, respuesta frente a la frustración que genera una figura de apego que no protege. La diferencia entre agresividad y violencia es que en el primer caso hay un reconocimiento del otro en la resolución de un conflicto, en cambio en la segunda se trata de dominar, de negar la integridad del otro (Berenstein, 1991). La violencia siempre implica una relación asimétrica. Tenemos que concluir que donde los afectos son más intensos, pueden surgir los sufrimientos más dolorosos.

Deberíamos estar atentos a todo tipo de violencia intrafamiliar, y en ella incluimos desde ya la violencia del género femenino hacia el masculino, que por estar todavía vigentes los estereotipos de la era patriarcal, no puede romperse la relación binaria ni asimétrica, pasando a ser con los años un espejo invertido de la violencia de género femenino a masculino que se va incrementando en nuestros días. Es indudable que las estadísticas no reflejan fielmente el sufrimiento del género masculino, ya que ambos géneros responden todavía a un conflicto de paradigmas de la Modernidad y de la Postmodernidad donde, como ya explicitamos, el hombre, como macho, no podía revelar el maltrato sufrido por su mujer. Es llamativo que todavía exista poca bibliografía sobre este tipo de violencia en relación con la violencia ejercida desde el hombre a la mujer. La riqueza de la investigación está en las entrevistas semidirigidas, donde las personas pueden explayarse en las situaciones que padecen en la cotidianeidad.

El incremento de la violencia de género femenino a masculino tal vez se deba al sojuzgamiento que padeció la mujer durante siglos, la represión del rol competitivo que fue aprendido y, ahora, frente a un lugar de cierta liberación y revalorización del lugar de la mujer en todos los ámbitos de la vida. Esto se despliega en las relaciones de pareja, con el signo de hostilidad y venganza por los años de sometimiento sufrido en las generaciones antecesoras. 
La problemática para investigar este fenómeno es que este tipo de violencia psicológica pertenece al ámbito privado. El varón no conoce en general los mecanismos legales para denunciarlos y, además, siente cierta vergüenza social. Esto también es un fenómeno que le ocurre a la mujer. Por eso, han sido tan relevantes las campañas sociales realizadas acerca de dar una cierta legitimidad a los fenómenos de violencia de género, a partir de los cuales la mujer se ha atrevido a denunciar públicamente su situación privativa en el ámbito familiar con su pareja. Con esto queremos significar que siempre la violencia doméstica, dado el carácter asimétrico del vínculo, genera en la víctima un sentimiento de culpa que hace que inhiba la denuncia pública. Esto provoca una mayor invisibilidad del fenómeno y un mayor sufrimiento psíquico por el silencio y el aislamiento que genera en la víctima.

A partir de las entrevistas denotamos que se trata de justificar la violencia de la mujer, al plantear que cuando un hombre padece una situación de violencia de género, es una respuesta de la mujer a una conducta del varón. Esto intenta negar y justificar la violencia ejercida por la mujer, legitimándola, ya que persisten los estereotipos del "sexo débil" y del "macho rudo".

La violencia presentada por Freud como la agresividad en juego entre los seres humanos fue objeto de múltiples reflexiones. En particular, pensamos que la más lograda es la que se encuentra en El Malestar en la Cultura.

el ser humano no es un ser manso, amable, a lo sumo capaz de defenderse si lo atacan, sino que es lícito atribuir a su dotación pulsional una buena cuota de agresividad. En consecuencia, el prójimo no es solamente un posible auxiliar y un objeto sexual, sino una tentación para satisfacer en él la agresión, explotar su fuerza de trabajo sin resarcirlo, usarlo sexualmente sin su consentimiento, desposeerlo de su patrimonio, humillarlo, infligirle dolores, martirizarlo y asesinarlo 'homo homini lupus'. Freud (1930)

Pasando ahora a otro aspecto de la violencia doméstica, es decir, la ejercida por los padres hacia los hijos, encontramos que el $16 \%$ de los encuestados considera que castigar a los hijos está justificado con la intención de educarlos mejor. En este caso, el castigo implica un grado de violencia física. Pensamos que el porcentaje parecería ser poco significativo, aunque evidentemente no erradicado, a pesar de que, en los tiempos que corren, socialmente parecería ser una conducta inapropiada. Por lo tanto, los hechos quedan reservados a la intimidad del hogar. Así es como el $13 \%$ manifiesta que sólo los padres pueden aplicar algún tipo de violencia física a los niños, y un $10 \%$ de la muestra afirma que algunos niños necesitan una palmada para obedecer y no les hace mal. Si bien la censura social opera con respecto a la violencia física en la crianza de los niños, resulta significativo que un $25 \%$ de los encuestados afirme que los padres que castigan físicamente a sus hijos lo hacen por "su bien". Estas respuestas, cruzadas con el material de entrevistas realizadas, nos hacen pensar que la violencia física hacia los niños no es una práctica social desechada dentro del ámbito familiar, a pesar de que es algo que también aparece silenciado en el ámbito público por el peso del discurso hegemónico del poder actual, ejercido por los profesionales de la salud y la educación. 


\section{Conclusiones}

Consideramos que la investigación descriptiva realizada por el Instituto de Ciencias Proyectuales de la Fundación UADE ha sido un intento de comenzar a hacer visible los diferentes tipos de violencia doméstica en nuestros días. Pensamos que estos fenómenos deberían traspasar el ámbito exclusivo de la clínica, ya que, por lo que comentamos, es difícil que, presentes dentro del seno familiar, se hagan públicas algunas modalidades vinculares que generan sufrimiento y menoscaban la subjetividad, por ser patrones vinculares que se viven como "normales". Generan una adaptación pasiva a la realidad cotidiana y su denuncia conlleva el temor de perder el hogar, fuente de sostén amoroso.

El entramado vincular de la intimidad de cada familia y/o pareja es naturalizado por sus miembros. Ya nos dice el refrán, al que tomamos como un saber popular, que "no hay que sacar los trapitos al sol". Así es como se silencia y se justifica la violencia doméstica ejercida por una figura a la que se adjudica cierto poder y que, asimismo, es idealizada por el resto de los miembros del grupo familiar. El temor a la pérdida de la seguridad y protección de los vínculos amorosos más íntimos (pareja, padres), hace que se genere una resistencia a hacer públicos estos sufrimientos y lleva hasta justificarlos por un sentimiento de culpa que se genera en las víctimas.

Estas cuestiones dan cuenta de lo difícil que resulta evaluar cuantitativamente una muestra, ya que los encuestados responden según la deseabilidad social, es decir, lo que creen que socialmente es esperable o correcto para los otros. Sin embargo, denotamos que cuando se realiza la triangulación de los datos cuantitativos con los cualitativos, gran parte de los participantes muestran que la violencia doméstica no está erradicada de los hogares y que no sólo se circunscribe a la efectuada por el hombre hacia la mujer.

Los cambios generados en las últimas décadas del género femenino han otorgado a la mujer una fortaleza e independencia psicológica (también económica) en las relaciones de pareja. El sufrimiento acaecido por las generaciones precedentes respecto al sojuzgamiento y estado de alienación subjetiva respecto del "hombre macho" ha quedado como marca identitaria. El odio reprimido en aquellas épocas que gestó conductas de abnegación y hasta de servidumbre por el grado de dependencia que la mujer tenía frente a "su hombre" quien era dueño y señor de sus vidas, hoy pareciera revertirse en conductas de venganza y menosprecio hacia el partenaire. La insatisfacción estructurante del deseo pareciera ser origen de reproches permanentes y actitudes violentas hacia el género masculino.

La mujer, que ya no necesita ser "la señora de ...", que todo lo puede sola: engendrar hijos (a partir de los avances científicos), sostenerse económicamente, obtener un lugar de reconocimiento (tanto profesional como social) y criar a sus hijos, muchas veces requiere que el hombre sea y actúe a imagen y semejanza de ella misma, sin 
permitirle al hombre aprender estas nuevas funciones a cumplir que no están en su acervo de aprendizajes previos. También le exige que no pierda su lugar de hombre protector, por lo que se observa que el género masculino está en crisis y no ha podido todavía realizar una adaptación activa a estas nuevas demandas de parte de su partenaire.

Pensamos que la violencia no es algo que puede erradicarse totalmente de la sociedad y tampoco del ámbito familiar. Sobre todo, si consideramos que vivimos en una sociedad hedonista e individualista donde prevalecen los vínculos narcisistas, en detrimento de los vínculos objetales que siempre generan frustraciones. Sin embargo, observamos que los hombres, más que las mujeres, tienden a tratar de conservar en nuestros tiempos la estructura familiar como fuente de apego y seguridad. Para el género femenino, los hijos representan fundamentalmente el sostén afectivo con prescindencia del varón.

Los hijos, criados en "libertad", padecen por otra parte ciertas conductas violentas por parte de sus padres tanto físicas como psicológicas. Los padres no toleran los procesos de aprendizaje ni tampoco las diferencias subjetivas que el niño presenta, quieren un hijo perfecto sin conflictos. Tratan de imponerles un modelo ideal paradójicamente con un mandato de libertad.

Consideramos también que la ausencia de ambos padres, que se produce cuando ambos tienen que cumplir con las exigencias profesionales, hace que se ausenten en el día a día del hogar y sustituyan esta carencia en la contención del vínculo parento/filial por jornadas diarias extensas con actividades extracurriculares. Pensamos que esta es otra forma de violencia que se produce justamente por ausencia y/o indiferencia afectiva del niño en desarrollo, no atendiendo a las necesidades evolutivas del hijo.

Concluimos que así como la violencia de género masculina hacia lo femenino ha generado la posibilidad de que muchas mujeres se sientan contenidas socialmente para denunciar públicamente un sufrimiento privado, es menester realizar campañas de concientización acerca de los diferentes tipos de violencia doméstica para que las personas, al sentir una cierta contención social y jurídica, puedan expresar estos padecimientos antes de llegar a situaciones patológicas más severas y hasta, algunas veces, sin salida. 


\section{Referencias Bibliográficas}

AULAGNIER,P. (1997). La violencia de la interpretación. Buenos Aires: Paidós.

---. (1994). Los destinos del placer.Buenos Aires: Paidós.

AUCIA, A., y otros. Grietas en el silencio. Una investigación sobre la violencia sexual, Rosario: Cladem-Insegnar. BERENSTEIN, I. (1991). Familia e inconciente.Buenos Aires: Paidós.

CAMPS, V. (1998). El siglo de las mujeres. Madrid: Cátedra.

CORSI. J. (1997). Violencia familiar Una mirada interdisciplinaria sobre un grave problema social. Buenos Aires: Paidós.

COTÉS BECHARELLI, E. (2000). El delito de malos tratos familiares. Nueva regulación. Madrid: Marcial Pons.

FISHER, H. (1999). El Primer Sexo. Las capacidades innatas de las mujeres y como están cambiando el mundo. Madrid: Taurus.

FRAISSE, G. (1993). «Del destino social al destino personal. Historia filosófica de la diferencia de los sexos». En Duby y Perrot, edit., Historia de las mujeres en Occidente. EI siglo XIX. Madrid: Taurus.

FREUD, S. (1986). EI Malestar en la Cultura Obras Completas, Tomo XXI. Buenos Aires: Amorrortu Editores,

GIL CALVO, E. (1997). El nuevo sexo débil. Los dilemas del varón postmoderno. Madrid: Temas de hoy.

GILMORE, D (1994). Hacerse hombre. Concepciones culturales de la masculinidad. Barcelona: Paidós.

QUIROGA, A. (1998). Crisis, procesos sociales, sujeto y grupo. Buenos Aires: Ediciones Cinco.

TOLDOS ROMERO, M. (2011). Hombres víctimas, mujeres agresoras: la cara oculta de la violencia entre sexos. México: Cántico. 